\title{
Properties of a Risk Measure Derived from Ruin Theory
}

\author{
Julien Trufin ${ }^{\mathrm{a}}$, Hansjoerg Albrecher ${ }^{\mathrm{b}}$ and Michel M. Denuit ${ }^{\mathrm{a}}$ \\ ${ }^{a}$ Institut de Statistique, Biostatistique et Sciences Actuarielles (ISBA), Universite Catholique \\ de Louvain, B-1348 Louvain-la-Neuve, Belgium. \\ ${ }^{\mathrm{b}}$ Ecole des HEC (Business School), University of Lausanne, Lausanne CH-1015, Switzerland.
}

This paper studies a risk measure inherited from ruin theory and investigates some of its properties. Specifically, we consider a value-at-risk (VaR)-type risk measure defined as the smallest initial capital needed to ensure that the ultimate ruin probability is less than a given level. This VaR-type risk measure turns out to be equivalent to the $\mathrm{VaR}$ of the maximal deficit of the ruin process in infinite time. A related Tail-VaR-type risk measure is also discussed.

The Geneva Risk and Insurance Review (2011) 36, 174-188. doi:10.1057/grir.2010.10; published online 24 August 2010

Keywords: ruin probability; classical risk model; value-at-risk (VaR); Tail-VaR; stochastic ordering

\section{Introduction and motivation}

The value-at-risk (VaR) quantifies how much an economic agent can expect to lose in 1 day, week, year, ... with a given probability. In today's financial world, VaR has become the benchmark risk measure. In insurance for instance, regulators often require that the available capital in the company, that is, the surplus of assets over liabilities (reserves), has at least to be equal to some appropriate VaR of the 1-year risk. This capital is used as a buffer against the risk that the premiums and reserves combined with the investment income turn out to be insufficient to cover future claims, making the probability that the insurer becomes technically insolvent small enough. We refer the reader, for example, to Volume 35, Issue 1 of the Geneva Papers on Risk and Insurance Issues and Practice for articles dealing with regulation and solvency for insurance companies. Also, readers interested in studies using the VaR concept could see, for example, Luciano and Kast (2001) for a derivation of insurance contracts which are Mean-VaR efficient, Huang (2006) and the references therein for a study of optimal insurance under a VaR constraint or Embrechts and Puccetti (2006) for the computation of VaR-based minimum capital requirement for a portfolio of operational risk losses. 
However, the appropriateness of risk measures defined over fixed time horizons usually advocated in both actuarial theory and practice can be questioned. It is not obvious that a time horizon of, say, 1 year for assessing the safety of a business activity is appropriate when uniformly applied to all companies (as is usual under the current regulation practice). Roughly speaking, the insurance risk over a horizon of 1 year of an insurance company is comparable with the one of a company with $n$ times as many policies (of the same kind) but a time horizon of $1 / n$ years. This becomes obvious when comparing the corresponding surplus processes. The presence and potential role of operational (or business) time as opposed to real (physical) time was already noticed by Filip Lundberg as early as 1903 in the ruin theory framework and may put into perspective the role of time in the context of risk measures. Lundberg's conclusion from this observation was that the actual assumptions behind the classical collective risk model are in fact less restrictive when time-invariant quantities like the infinite-time ruin probability are considered (in particular, from this perspective the assumption of having a constant Poisson intensity in the model which will appear later in the paper can be weakened without changing the results). The choice of an appropriate time horizon for measuring risk as a function of the size of the company is not the topic of this paper, but this aspect gives additional motivation to investigate both the potential and the limits of time-invariant measures of solvency risk and to investigate ruin theory concepts as measurement tools in the framework of risk measures.

Whereas VaR is usually defined in terms of a given time horizon, as explained above, in certain applications it may be more natural to look for measures that give a more robust reflection of the risk inherent in a business activity in a random environment. In particular, the VaR for a given time horizon does not reflect the possible adverse financial situations in between or beyond the specified time interval. On the other hand, in an insurance context, starting with the work of Filip Lundberg and Harald Cramér in the early 20th century, ruin theory has always studied somewhat robust measures of insurance risk. In particular, the probability of ruin can be interpreted as the continuous alternative to the VaR.

One historical purpose of studying ruin probabilities was to obtain the amount of initial capital needed to guarantee a certain probability of solvency throughout the lifetime of the process, given that both the premium strategy and the aggregate claim process are stationary over time. In that sense the ruin probability might be interpreted as a measure of dynamic risk in a static environment (meaning that the initial capital and the premium strategy are fixed and later on one is not able to or does not want to interfere during the lifetime of the process). In particular, the correspondingly allocated capital will then suffice to cope with the involved insurance risk at all times according to 
some acceptance level of $\varepsilon$. It is this type of robustness that makes the ruin probability still nowadays an interesting quantity in this context. Most of all, the type of thinking coming from ruin theory is often considered important by practitioners.

The purpose of this paper is to consider certain properties of risk measures that are motivated by ruin theory. The goal is not so much to advocate them for practical use in risk management, but rather to improve the understanding of the connections between these fields and to see whether the quite active research field of ruin theory can offer additional insight for people dealing with practical risk management of insurance companies as well.

This paper discusses risk measures for insurance portfolios, which are derived from actuarial ruin theory. Dhaene et al. (2003) give ample motivation for an exponential risk measure inherited from the Cramér-Lundberg upper bound for the ruin probability in a discrete-time ruin model. Cheridito et al. (2006) mention (in an application of their study on coherent risk measures for unbounded stochastic processes) a VaR-type risk measure based on the infinite-time ruin probability itself. In general, over the last years the relative position and relation between (insurance) risk measures that fulfil a certain list of axioms on the one hand, and classical tools in ruin theory to assess the riskiness of an insurance activity in the collective framework on the other hand, has often been a matter of debate. In this paper, we would like to take up this issue and investigate in more detail some properties of the VaR-type risk measure based on the ruin probability that is mentioned in Cheridito et al. (2006). For ease of exposition, we will restrict the considerations to the classical compound Poisson process. Note that Geman (1998) also emphasized the usefulness of the actuarial ruin paradigm and Embrechts et al. (2004) demonstrated that ruin theory can be especially useful for operational risk.

Our paper is organized as follows. In the following section, we set up the scene by recalling some properties and tools of the compound Poisson risk model. In the subsequent section, we derive various properties of a VaR-type risk measure, which is defined as the smallest amount of capital needed to ensure a ruin probability below a given probability level. In the penultimate section, we then discuss the corresponding Tail-VaR-type risk measure. The final section concludes.

\section{Risk model and harmonic mean residual life order}

\section{Compound Poisson surplus model}

This section recalls some basic results about ruin probabilities, which will be useful in our analysis. For more details, we refer the interested reader, 
for example, to Asmussen (2000). The surplus process (or risk process) is defined as

$$
U_{t}=u+c t-S_{t}, \quad t \geqslant 0,
$$

where $U_{t}$ is the insurer's capital at time $t$ starting from some initial capital $U_{0}=u, c$ is the (constant) premium income per unit of time and $S_{t}=\sum_{k=1}^{N_{t}} X_{k}$ is the total claim amount up to time $t$, with $N_{t}$ the corresponding number of claims, and $X_{k}$ the size of the $k$ th claim, assumed to be non-negative. The claim number process $\left\{N_{t}, t \geqslant 0\right\}$ is assumed to be Poisson with constant rate $\lambda$. The $X_{k} \mathrm{~s}$ are independent and distributed as $X$, with distribution function $F_{X}$. They are furthermore assumed to be independent of $\left\{N_{t}, t \geqslant 0\right\}$. We assume that the premium rate is of the form $c=(1+\eta) \lambda \mathbb{E}[X]$ where $\eta>0$ is called the safety loading. Henceforth, $\eta$ is assumed to be the same for all the risk processes used in this paper.

The ruin time $T$ is defined as

$$
T=\left\{\begin{array}{l}
\min \left\{t \geqslant 0 \mid U_{t}<0\right\} \\
+\infty \quad \text { if } U_{t} \geqslant 0 \text { for all } t,
\end{array}\right.
$$

and the ruin probability is $\psi(u)=\operatorname{Pr}[T<\infty]$. If needed, we will denote the ruin probability as $\psi_{X}$ to make explicit the dependence on $X$. Let $L_{t}=S_{t}-c t$ be the aggregate loss at time $t$, that is the amount by which the insurance claims exceed the collected premiums at some time $t$, the ruin time $T$ can also be expressed as

$$
T=\min \left\{t \geqslant 0 \mid u-L_{t}<0\right\} .
$$

Correspondingly, $\psi(u)=\operatorname{Pr}[L>u]$, where $L=\max _{t \geqslant 0} L_{t}$ denotes the maximal aggregate loss of the process.

\section{Compound geometric representation of the ruin probability}

It is well-known that $\psi$ coincides with the tail function of a compound geometric distribution since $L$ can be decomposed as

$$
L=\sum_{j=1}^{M} D_{j}
$$

where $M$ follows the geometric distribution with success probability $1-\psi(0)=$ $\eta / 1+\eta$ and where $D_{1}, D_{2}, \ldots$ are the ladder heights of the loss process which are independent and identically distributed. In the compound Poisson model, the 
common distribution function of the $D_{j}$ s is given by the integrated tail distribution

$$
F_{D}(y)=\int_{0}^{y} \frac{1-F_{X}(x)}{\mathbb{E}[X]} d x, \quad y>0 .
$$

The non-ruin probability is then given by

$$
1-\psi(u)=\sum_{m=0}^{\infty} p(1-p)^{m} F_{D}^{*(m)}(u),
$$

where $p=\eta / 1+\eta$ and $F_{D}^{*(m)}$ is the $m$-fold convolution of $F_{D}$.

Note that the mapping $F_{X} \rightarrow F_{D}$ is well known in applied probability, where (apart from the name integrated tail distribution) $F_{D}$ is often called the stationary forward recurrence time, the equilibrium distribution or the residual lifetime in the literature. The relationship between the moments of $X$ and of $D$ is given by

$$
\mathbb{E}\left[D^{k}\right]=\frac{\mathbb{E}\left[X^{k+1}\right]}{(k+1) \mathbb{E}[X]}, \quad k=1,2, \ldots
$$

An important result in our setting is as follows. Recall the definition of the stochastic dominance and of the convex order. Given two random variables $X$ and $Y, X$ precedes $Y$ in the stochastic dominance, denoted as $X \preceq_{\text {st }} Y$, if the inequality $\mathbb{E}[g(X)] \leqslant \mathbb{E}[g(Y)]$ holds for any non-decreasing function $g$ such that the expectations exist. Similarly, $X$ precedes $Y$ in the convex order, denoted as $X \preceq_{\mathrm{cx}} Y$, if the inequality $\mathbb{E}[g(X)] \leqslant \mathbb{E}[g(Y)]$ holds for any convex function $g$ such that the expectations exist. Then, denoting as $F_{E}$ the integrated tail distribution associated to $F_{Y}$, and as $E$ (resp. $Y$ ) a random variable with distribution function $F_{E}$ (resp. $F_{Y}$ ), we have

$$
X \preceq_{\mathrm{cx}} Y \Rightarrow D \preceq_{\mathrm{st}} E .
$$

Henceforth, we will also need the increasing convex, or stop-loss, order defined as follows. Given two random variables $X$ and $Y, X$ precedes $Y$ in the increasing convex order, denoted as $X \preceq_{\text {icx }} Y$, if the inequality $\mathbb{E}[g(X)] \leqslant \mathbb{E}[g(Y)]$ holds for any non-decreasing convex function $g$ such that the expectations exist.

\section{Harmonic mean residual life order}

Michel (1987) defined a stochastic order relation among claim size distributions by reference to the comparison of ruin probabilities. This order in fact compares the ladder-height distribution $F_{D}$ of the renewal process describing 
claim occurrences. The harmonic mean residual life order, which proves useful in reliability applications, naturally arises in this context. For other applications of this ordering in actuarial science, we refer the interested reader, for example, to Lefèvre and Utev (2001).

Given two non-negative random variables $X$ and $Y$, recall that $X$ is said to be smaller than $Y$ in the harmonic mean residual life order (denoted as $X \preceq_{\mathrm{hmrl}} Y$ ) when

$$
\frac{\int_{t}^{\infty} \bar{F}_{X}(u) d u}{\mathbb{E}[X]} \leqslant \frac{\int_{t}^{\infty} \bar{F}_{Y}(u) d u}{\mathbb{E}[Y]} \text { for all } t \geqslant 0,
$$

where $\bar{F}_{X}=1-F_{X}$ and $\bar{F}_{Y}=1-F_{Y}$. The inequality in Eq. (7) can be equivalently written as

$$
\frac{\mathbb{E}\left[(X-t)_{+}\right]}{\mathbb{E}[X]} \leqslant \frac{\mathbb{E}\left[(Y-t)_{+}\right]}{\mathbb{E}[Y]} \text { for all } t \geqslant 0 .
$$

From Eq. (8) it follows that $X \preceq_{\mathrm{hmrl}} Y$ if, and only if,

$$
\frac{\mathbb{E}[g(X)]}{\mathbb{E}[X]} \leqslant \frac{\mathbb{E}[g(Y)]}{\mathbb{E}[Y]}
$$

for all non-decreasing convex functions $g: \mathbb{R}^{+} \rightarrow \mathbb{R}$, provided the expectations exist.

A useful property for the present study is Theorem 2.B.16 in Shaked and Shanthikumar (2007). It shows that any mixture of two distributions ordered in the $\varsigma_{\mathrm{hmrl}}$ order is bounded from above and from below by the components of the mixture. Formally, let $X$ and $Y$ be such that $X \preceq_{\mathrm{hmrl}} Y$ and define the distribution function $F_{Z}=\beta F_{X}+(1-\beta) F_{Y}$ for some $\beta \in(0,1)$. Then,

$$
X \preceq_{\mathrm{hmrl}} Z \preceq_{\mathrm{hmrl}} Y \text {. }
$$

Remark: Neither of the orders $\preceq_{\text {st }}$ and $\preceq_{\text {hmrl }}$ implies the other. It can nevertheless be shown that

$$
X \preceq_{\mathrm{hmrl}} Y \Rightarrow \mathbb{E}[X \mid X>0] \leqslant \mathbb{E}[Y \mid Y>0],
$$

so that when $X$ and $Y$ are positive almost surely

$$
X \preceq_{\mathrm{hmrl}} Y \Rightarrow \mathbb{E}[X] \leqslant \mathbb{E}[Y] .
$$


If $\mathbb{E}[X]=\mathbb{E}[Y]$ then Eq. (9) ensures that

$$
X \preceq_{\mathrm{hmrl}} Y \Leftrightarrow X \preceq_{\mathrm{cx}} Y .
$$

\section{VaR-type risk measure}

Whereas in Dhaene et al. (2003), essentially the risk measure is the necessary annual premium amount such that for a given initial capital $u$ the resulting ruin probability is bounded by $\varepsilon$, in this paper we would like to focus on another approach linking ruin probabilities with the framework of risk measures. We consider the continuous-time risk model (1) and, instead of fixing $u$, we assume that the safety loading $\eta$ is fixed and ask for the amount of initial capital needed in order to bound the ruin probability by $\varepsilon$. That is, we define now the ruin-consistent VaR risk measure as

$$
\rho_{\varepsilon}[X]=\inf \{v \geqslant 0 \mid \psi(v) \leqslant \varepsilon\}=\psi^{-1}(\varepsilon) .
$$

In words, $\rho_{\varepsilon}[X]$ is the smallest amount of capital needed such that the ultimate ruin probability $\psi$ for a risk process with individual claim sizes distributed as $X$, is at most equal to some specified probability level $\varepsilon$. Working directly on the basis of the ruin probability $\psi$ (instead of on the basis of Lundberg's upper bound used by Dhaene et al. (2003)) has the advantage that the risk measure is exact rather than a bound and second, and this is also quite important, the method is then not restricted to insurance risk processes with exponentially bounded claims for which a Lundberg exponent exists. In particular, many realistic descriptions of risk processes are based on heavytailed (subexponential) claims for which the Lundberg exponent does not exist.

One possible interpretation of this approach is as follows. Competition among companies (market pressure, underwriting cycles, etc.) determines a safety loading $\eta$ that can be realized in the insurance market and then $\rho_{\varepsilon}[X]$ reflects the amount of capital needed to ensure a stable business according to the safety level $\varepsilon$, assuming "stationary" insurance business (the amount $\rho_{\varepsilon}[X]$ then puts the portfolio into an acceptable position with respect to the safety measure $\psi$ ). So in this approach the premium income is considered to be (the market-induced "balancing") part of the insurance risk in the portfolio rather than the control variable. Measuring risk by the infinite-time ruin probability $\psi$ entails that we deal with an unbounded time horizon. Hence, an acceptable position in such a framework reflects long-term stability thinking. Naturally, as time evolves, using claim experience and further acquired information, the capital requirements will be readjusted. But compared to the classical 1-year time horizon $\mathrm{VaR}$ approach, in the above setup the incentive is to always maintain a (with respect to available information) sustainable strategy. It is 
worth to mention that the vast majority of the results in risk theory consider $\eta$ as fixed when the effect of switching from one severity distribution to another is studied.

Remark: It may appear surprising that the risk measure $\rho_{\varepsilon}$ does not explicitly depend on the claim frequency $\lambda$. This is because the ruin probability itself does not depend on $\lambda$ as we are allowed to switch to any operational time scale. Formally, this is clear from the compound geometric representation in Eq. (6). This means that $\rho_{\varepsilon}$ is not influenced by the volume of the portfolio reflected in $\lambda$, since the fixed value of $\eta$ ensures that the corresponding value of $c$ compensates for the change in the portfolio volume. This is because the classical risk model values "time diversification". Hence, the number of policies does not matter, but well the distribution of the claim amount they generate, that is $\rho_{\varepsilon}$ only depends on $X$ (and on $\eta$ ). Depending on the field of application, this may be considered as an advantage or disadvantage for the applicability of $\rho_{\varepsilon}$.

Another way to look at $\rho_{\varepsilon}$ is to consider it as a VaR applied to the transformed risk $L$ (the maximal aggregate loss). Specifically, for any probability level $\varepsilon$,

$$
\rho_{\varepsilon}[X]=F_{L}^{-1}(1-\varepsilon)=\inf \left\{t \geqslant 0 \mid F_{L}(t) \geqslant 1-\varepsilon\right\} .
$$

Instead of taking the $\mathrm{VaR}$ of the claim severity $X$, we first transform $X$ into $L$ and then compute the VaR of this geometric compound sum. The question now is to determine whether this is a safe strategy, that is, whether $L$ is more dangerous than $X$, in some sense. To study this problem, let us decompose the switch from $X$ to $L$ in two steps: (i) a change from $F_{X}$ to the corresponding integrated tail distribution $F_{D}$ followed by (ii) a geometric compounding.

Step (i): Recall that a random variable is said to be NWUE (or New Worse than Used in Expectation) if $\mathbb{E}\left[(X-t)_{+}\right] \geqslant \mathbb{E}[(X)] \bar{F}_{X}(t)$ for all $t \geqslant 0$. This basically means that $X$ dominates the Negative Exponential with the same expectation in the $\preceq_{\mathrm{cx}}$ sense. Many standard claim size distributions fulfil this property (for appropriate values of their parameters). According to Theorem 1.A.31 in Shaked and Shanthikumar (2007), we have that $X$ NWUE $\Rightarrow X \preceq_{\mathrm{st}} D$. Hence, step (i) in the switch from $X$ to $L$ appears to be conservative since we replace $X$ with a "larger" $D$.

Step (ii): The second step consists in replacing $D$ with $\sum_{j=1}^{M} D_{j}$, where the $D_{j} s$ are independent copies of $D$. Assume that $\eta \in(0,1)$ so that $\mathbb{E}[M]>1$ and $1 \preceq_{\text {icx }} M$. As a $\preceq_{\text {icx }}$-ranking of the number of terms induces the same ordering for the resulting compound sums, we get $D \preceq_{\text {icx }} L$ so that Step (ii) is also conservative. Combining the two steps reveals that $X \preceq_{\text {icx }} L$ holds, so that the risk $X$ is first replaced with a more dangerous (in the $\preceq_{\text {icx }}$-sense) random variable $L$ before taking a VaR. 
Properties of $\rho_{\varepsilon}$

The risk measure $\rho_{\varepsilon}$ possesses the following properties.

Property 3.1

(i) The risk measure $\rho_{\varepsilon}$ is positively homogeneous, that is, $\rho_{\varepsilon}[a X]=a \rho_{\varepsilon}[X]$ for any constant $a>0$.

(ii) The risk measure $\rho_{\varepsilon}$ agrees with the stop-loss order, that is $X \preceq_{\text {icx }} Y \Rightarrow$ $\rho_{\varepsilon}[X] \leqslant \rho_{\varepsilon}[Y]$.

(iii) If $Y$ and $Z$ are identically distributed and if $Z$ is more positively dependent on $X$ than $Y$, in the sense that the inequality $\operatorname{Pr}[X \leqslant x, Y \leqslant y] \leqslant \operatorname{Pr}[X \leqslant x$, $Z \leqslant y]$ holds for all $x$ and $y$, then the risk measure $\rho_{\varepsilon}$ expresses the fact that $X+Z$ is more dangerous than $X+Y$ as $\rho_{\varepsilon}[X+Y] \leqslant \rho_{\varepsilon}[X+Z]$.

(iv) If $Z$ is a mixture of $X, Y$ and $X+Y$ (that is, $F_{Z}=p_{1} F_{X}+p_{2} F_{Y}+p_{3} F_{X+Y}$ with $p_{i} \geqslant 0$ for $i=1,2,3$ and $\left.\sum_{i=1}^{3} p_{i}=1\right)$ such that $\alpha_{\varepsilon} Z \preceq_{\text {hmrl }} Y$, where $\alpha_{\varepsilon}=\rho_{\varepsilon}[Y] / \rho_{\varepsilon}[X]+\rho_{\varepsilon}[Y]$, then $\rho_{\varepsilon}[Z] \leqslant \rho_{\varepsilon}[X]+\rho_{\varepsilon}[Y]$.

Proof Item (i) is obvious since multiplying the annual claim amount by $a$ has the same effect on the annual premium income, so that the initial capital needed to have an ultimate ruin probability of $\varepsilon$ is also multiplied by $a$. Formally, replacing $X$ with $a X$ changes $L$ into $a L$ which results in $a \rho_{\varepsilon}[X]$. Considering item (ii), we know that $X \preceq_{\text {icx }} Y \Rightarrow \psi_{X}(u) \leqslant \psi_{Y}(u)$ for all $u$. This classical result of risk theory can be found, for example, in standard textbooks as Kaas et al. (2008, Section 7.4.2). Next, item (iii) is deduced from (ii) since $X+Y \preceq_{\mathrm{cx}} X+Z$. Finally, for item (iv), coming back to the compound geometric representation of the ruin probability, we can write

$$
\alpha_{\varepsilon} Z \preceq \text { hmrl } Y \Rightarrow \psi_{\alpha_{\varepsilon} Z}\left(\rho_{\varepsilon}[Y]\right) \leqslant \psi_{Y}\left(\rho_{\varepsilon}[Y]\right) .
$$

Now, multiplying the claim sizes by a fixed coefficient $\alpha_{\varepsilon}$ is equivalent to dividing the initial capital by the same factor. Hence,

$$
\psi_{Z}\left(\rho_{\varepsilon}[X]+\rho_{\varepsilon}[Y]\right)=\psi_{Z}\left(\frac{\rho_{\varepsilon}[Y]}{\alpha_{\varepsilon}}\right)=\psi_{\alpha_{\varepsilon} Z}\left(\rho_{\varepsilon}[Y]\right) \leqslant \psi_{Y}\left(\rho_{\varepsilon}[Y]\right) \leqslant \varepsilon
$$

so that the inequality $\rho_{\varepsilon}[Z] \leqslant \rho_{\varepsilon}[X]+\rho_{\varepsilon}[Y]$ indeed holds true, which completes the proof.

In particular, it is clear, from (iii), that $\rho_{\varepsilon}$ is comonotonicity-consistent (in the terminology of Dhaene et al. (2003)). Furthermore, from (iv), it also 
follows that $\rho_{\varepsilon}$ recognizes diversification effect under conditions of exchangeable risks, negative quadrant dependent risks with equivalent sizes, and risks that are members of scale family distributions, whatever the dependence between them. Let us formally establish these results.

\section{Property 3.2}

(i) The risk measure $\rho_{\varepsilon}$ is subadditive for exchangeable risks, that is, $\rho_{\varepsilon}[X+Y] \leqslant \rho_{\varepsilon}[X]+\rho_{\varepsilon}[Y]$ if $X$ and $Y$ satisfy $\operatorname{Pr}\left[X \leqslant t_{1}, Y \leqslant t_{2}\right]=\operatorname{Pr}\left[X \leqslant t_{2}\right.$, $\left.Y \leqslant t_{1}\right]$ for all $t_{1}$ and $t_{2}$. This is in particular the case if $X$ and $Y$ are independent and identically distributed.

(ii) If $X$ and $Y$ are negatively quadrant dependent and identically distributed, that is, the inequality $\operatorname{Pr}[X \leqslant x, Y \leqslant y] \leqslant \operatorname{Pr}[X \leqslant x] \operatorname{Pr}[Y \leqslant y]$ holds for all $x$ and $y$ then the risk measure $\rho_{\varepsilon}$ is subadditive, that is, $\rho_{\varepsilon}[X+Y] \leqslant$ $\rho_{\varepsilon}[X]+\rho_{\varepsilon}[Y]$.

(iii) If $X=\beta V_{1}$ and $Y=\gamma V_{2}$, where $V_{1}$ and $V_{2}$ are identically distributed as $V$, then the risk measure $\rho_{\varepsilon}$ is subadditive, that is, $\rho_{\varepsilon}[X+Y] \leqslant \rho_{\varepsilon}[X]+\rho_{\varepsilon}[Y]$.

Proof Let us consider Property 3.1 (iv) with $p_{1}=p_{2}=0$. Then, as $\alpha_{\varepsilon}=1 / 2$, item (i) is obvious since $(X+Y) / 2 \preceq_{\mathrm{cx}} Y$. Next, considering (ii), since $\alpha_{\varepsilon}=1 / 2$, it suffices to note that $(X+Y) / 2 \preceq_{\mathrm{cx}}\left(X^{\perp}+Y^{\perp}\right) / 2 \preceq_{\mathrm{cx}} Y$, where $\left(X^{\perp}, Y^{\perp}\right)$ has independent components with the same marginals as $(X, Y)$. Finally, for item (iii), we have to show that $\beta V_{1}+\gamma V_{2} \preceq_{\mathrm{cx}}(\beta+\gamma) V$, since $\alpha_{\varepsilon}=\gamma / \beta+\gamma$. Now, as $\operatorname{Pr}[\beta V \leqslant x, \gamma V \leqslant y] \geqslant \operatorname{Pr}\left[\beta V_{1} \leqslant x, \gamma V_{2} \leqslant y\right]$ holds for all $x$ and $y$, we have that, by equation 9.A.19 in Shaked and Shanthikumar (2007), $\beta V_{1}+\gamma V_{2} \preceq_{\mathrm{cx}}$ $(\beta+\gamma) V$.

\section{Application in terms of portfolios}

Now, let us define the two processes $S_{t}^{(1)}=\sum_{i=1}^{N_{t}^{(1)}+N_{t}} X_{i}$ and $S_{t}^{(2)}=\sum_{i=1}^{N_{t}^{(2)}+N_{t}} Y_{i}$ where $\left\{N_{t}, t \geqslant 0\right\},\left\{N_{t}^{(1)}, t \geqslant 0\right\}$ and $\left\{N_{t}^{(2)}, t \geqslant 0\right\}$ are independent Poisson processes with constant rates $\lambda, \lambda_{1}$ and $\lambda_{2}$ respectively, and the $X_{k}$ s (respectively $\left.Y_{k} \mathrm{~s}\right)$ are independent and distributed as the generic random variable $X$ (respectively $Y$ ). Furthermore, $X_{k} \mathrm{~s}$ and $Y_{k}$ s are assumed to be independent of $\left\{N_{t}, t \geqslant 0\right\},\left\{N_{t}^{(1)}, t \geqslant 0\right\}$ and $\left\{N_{t}^{(2)}, t \geqslant 0\right\}$. Then, the properties of $\rho_{\varepsilon}$ allow us to deduce some cases where merging two portfolios with cumulative losses described by $S_{t}^{(1)}$ and $S_{t}^{(2)}$ is beneficial.

Assume that the $X_{k} \mathrm{~s}$ are independent of the $Y_{k} \mathrm{~s}$. Then, $S_{t}^{(1)}+S_{t}^{(2)}$ is a compound Poisson process with intensity $\lambda+\lambda_{1}+\lambda_{2}$ and generic claim size $Z$ with distribution function $F_{Z}=p_{1} F_{X}+p_{2} F_{Y}+p_{3} F_{X+Y}$, where $p_{1}=\lambda_{1} /\left(\lambda_{1}+\lambda_{2}+\lambda\right)$, $p_{2}=\lambda_{2} /\left(\lambda_{1}+\lambda_{2}+\lambda\right)$ and $p_{3}=\lambda /\left(\lambda_{1}+\lambda_{2}+\lambda\right)$. Hence, if $\alpha_{\varepsilon} Z \preceq_{\text {hmrl }} Y$, then, by Property 3.1 (iv), a diversification effect at level $\varepsilon$ is recognized. The next result gives an illustration. 
Proposition 3.3 Let $X=\beta V_{1}$ and $Y=\gamma V_{2}$, with $\beta \leqslant \gamma$, where $V_{1}$ and $V_{2}$ are independent and identically distributed. Then $\alpha_{\varepsilon} Z \preceq_{\mathrm{hmrl}} Y$.

Proof Obviously, we have $X \preceq_{\mathrm{hmrl}} Y \Leftrightarrow \beta \leqslant \gamma$. Hence, it is easily seen from Eq. (8) that $\alpha_{\varepsilon} X \preceq_{\mathrm{hmrl}} Y$ and $\alpha_{\varepsilon} Y \preceq_{\mathrm{hmrl}} Y$. Now, we also know by the proof of Property 3.2 (iii) that $\alpha_{\varepsilon}(X+Y) \preceq_{\mathrm{hmrl}} Y$. Hence, we necessarily have $\alpha_{\varepsilon} Z \preceq_{\mathrm{hmrl}} Y$. Indeed,

$$
\begin{aligned}
\frac{\mathbb{E}\left[\left(\alpha_{\varepsilon} Z-t\right)_{+}\right]}{\mathbb{E}\left[\alpha_{\varepsilon} Z\right]}= & \frac{\mathbb{E}\left[\left(\alpha_{\varepsilon} X-t\right)_{+}\right] p_{1}+\mathbb{E}\left[\left(\alpha_{\varepsilon} Y-t\right)_{+}\right] p_{2}+\mathbb{E}\left[\left(\alpha_{\varepsilon}(X+Y)-t\right)_{+}\right] p_{3}}{\mathbb{E}\left[\alpha_{\varepsilon} X\right] p_{1}+\mathbb{E}\left[\alpha_{\varepsilon} Y\right] p_{2}+\mathbb{E}\left[\alpha_{\varepsilon}(X+Y)\right] p_{3}} \\
= & \frac{\frac{\mathbb{E}\left[\left(\alpha_{\varepsilon} X-t\right)_{+}\right]}{\mathbb{E}\left[\alpha_{\varepsilon} X\right]} \mathbb{E}\left[\alpha_{\varepsilon} X\right] p_{1}+\frac{\mathbb{E}\left[\left(\alpha_{\varepsilon} Y-t\right)_{+}\right]}{\mathbb{E}\left[\alpha_{\varepsilon} Y\right]} \mathbb{E}\left[\alpha_{\varepsilon} Y\right] p_{2}+\frac{\mathbb{E}\left[\left(\alpha_{\alpha}(X+Y)-t\right)_{+}+\right]}{\mathbb{E}\left[\alpha_{\varepsilon}(X+Y)\right]} \mathbb{E}\left[\alpha_{\varepsilon}(X+Y)\right]_{p_{3}}}{\mathbb{E}\left[\alpha_{\varepsilon} X\right] p_{1}+\mathbb{E}\left[\alpha_{\varepsilon} Y\right] p_{2}+\mathbb{E}\left[\alpha_{\varepsilon}(X+Y)\right] p_{3}} \\
& \leqslant \frac{\frac{\mathbb{E}\left[(Y-t)_{+}\right]}{\mathbb{E}[Y]}\left(\mathbb{E}\left[\alpha_{\varepsilon} X\right] p_{1}+\mathbb{E}\left[\alpha_{\varepsilon} Y\right] p_{2}+\mathbb{E}\left[\alpha_{\varepsilon}(X+Y)\right] p_{3}\right)}{\mathbb{E}\left[\alpha_{\varepsilon} X\right] p_{1}+\mathbb{E}\left[\alpha_{\varepsilon} Y\right] p_{2}+\mathbb{E}\left[\alpha_{\varepsilon}(X+Y)\right] p_{3}} \\
= & \frac{\mathbb{E}\left[(Y-t)_{+}\right]}{\mathbb{E}[Y]}
\end{aligned}
$$

which ends the proof.

Assume that $\lambda=0$. Hence, $S_{t}^{(1)}$ and $S_{t}^{(2)}$ are independent. Then, in this particular case, we have the following result.

Proposition 3.4 Assume that $\lambda=0$. If $X \preceq_{\mathrm{hmrl}} Y$, then $\alpha_{\varepsilon} Z \preceq_{\mathrm{hmrl}} Y$.

Proof From Eq. (10), we have that $X \preceq_{\mathrm{hmrl}} Y \Rightarrow Z \preceq_{\mathrm{hmrl}} Y$. Also, it is easily seen from Eq. (8) that $\alpha_{\varepsilon} Z \preceq_{\mathrm{hmrl}} Z$ so that $X \preceq_{\mathrm{hmrl}} Y \Rightarrow \alpha_{\varepsilon} Z \preceq_{\mathrm{hmrl}} Y$.

Let us give some examples taken from Heilmann and Schröter (1991) where $X \preceq_{\mathrm{hmrl}} Y$ holds true:

(1) If $X$ is Uniformly distributed over the interval $(a, b)$ and $Y$ is Uniformly distributed over the interval $\left(a^{\prime}, b^{\prime}\right)$, then $X \preceq_{\mathrm{hmrl}} Y \Leftrightarrow a+b \leqslant a^{\prime}+b^{\prime}$ and $b \leqslant b^{\prime}$.

(2) If $X$ is Exponentially distributed with mean $1 / a$ and $Y$ is Exponentially distributed with mean $1 / a^{\prime}$ then $X \preceq_{\mathrm{hmrl}} Y \Leftrightarrow a \geqslant a^{\prime}$.

(3) If $X$ is Pareto distributed with parameters $a$ and $b$, that is, $X$ has distribution function $1-(a / x+a)^{b}, x>0$, and if $Y$ is Pareto distributed with parameters $a^{\prime}$ and $b^{\prime}$ with $\min \left(b, b^{\prime}\right)>1$, then $X \preceq_{\text {hmrl }} Y \Leftrightarrow \frac{b-1}{b^{\prime}-1} \geqslant \max \left(\frac{a}{a^{\prime}}, 1\right)$

Notice that the second example is a particular case of the equivalence $X \preceq_{\text {hmrl }} Y \Leftrightarrow \beta \leqslant \gamma$, where $X=\beta V_{1}$ and $Y=\gamma V_{2}$, with $V_{1}$ and $V_{2}$ are independent and identically distributed. 


\section{Tail-VaR-type risk measure}

Finally, we would like to point out that a natural extension of $\rho_{\varepsilon}$ is the TailVaR (or average VaR) defined as

$$
\bar{\rho}_{\varepsilon}[X]=\frac{1}{\varepsilon} \int_{0}^{\varepsilon} \rho_{w}[X] d w,
$$

where $\rho_{w}$ is the VaR-type risk measure at level $w$ discussed in the previous section. By definition we can write

$$
\bar{\rho}_{\varepsilon}[X]=\frac{1}{\varepsilon} \int_{0}^{\varepsilon} \psi^{-1}(w) d w,
$$

where $\psi^{-1}(w)$ is the inverse function of the ruin probability, or further

$$
\bar{\rho}_{\varepsilon}[X]=\frac{1}{\varepsilon} \int_{0}^{\varepsilon} F_{L}^{-1}(1-w) d w .
$$

Hence, $\bar{\rho}_{\varepsilon}[X]$ appears to be the Tail-VaR of $L$. Under the conditions discussed above, we now have

$$
X \preceq_{\mathrm{icx}} L \Rightarrow \operatorname{TVaR}[X ; \varepsilon] \leq \operatorname{TVaR}[L ; \varepsilon]=\bar{\rho}_{\varepsilon}[X] .
$$

Alternatively one can rewrite

$$
\begin{aligned}
\bar{\rho}_{\varepsilon}[X] & =\frac{1}{\varepsilon} \int_{\psi^{-1}(0)}^{\psi^{-1}(\varepsilon)} w d \psi(w) \\
& =\psi^{-1}(\varepsilon)+\frac{1}{\varepsilon} \int_{0}^{\varepsilon} \frac{w d w}{\left|\psi^{\prime}\left(\psi^{-1}(w)\right)\right|} \\
& =\psi^{-1}(\varepsilon)+\frac{1}{\varepsilon} \mathbb{E}\left[\left(L-\psi^{-1}(\varepsilon)\right)_{-}\right] \\
& =\mathbb{E}\left[L \mid L>F_{L}^{-1}(1-\varepsilon)\right] .
\end{aligned}
$$

The last two equalities follow from general properties of Tail-VaR, as, for example, given in Denuit et al. (2005, Section 2.4) or in Pflug and Römisch (2007). 
186

From the above it becomes clear that the measure $\bar{\rho}_{\varepsilon}[X]$ represents the amount of capital needed to be able to cope "in expectation" also with the insurance loss in those problematic cases that occur with probability less than $\varepsilon$. One also observes that explicit knowledge of the ruin probability expression can tremendously simplify the calculations of this risk measure. In Cheridito et al. (2006) it is shown that $\bar{\rho}_{\varepsilon}[X]$ is coherent and hence satisfies several desirable properties. However, on a computational level, $\bar{\rho}_{\varepsilon}$ will typically be harder to deal with than $\rho_{\varepsilon}$ and, as often in related contexts, in practice there will be a trade-off between the gain in theoretical attractiveness and the lowering of practicability of the resulting expressions when choosing feasible measures for the insurance risk.

\section{Conclusion}

In this paper, we discussed the classical concept of the ruin probability of an insurance portfolio in the framework of risk measures and established some properties of the corresponding naturally motivated VaR-type risk measure $\rho_{\varepsilon}$. The crucial assumption in this approach is that the collective insurance business in the portfolio has a certain degree of stationarity and that the safety loading is fixed (for instance, determined by market conditions). Note that apart from that the results are quite general as they are expressed through the ruin probability directly. This may for instance be particularly welcome in situations with dependence among the insurance risks for which still some results on the ruin probability are available. Finally, we would like to emphasize once more that the purpose of this paper is not to advocate the practical use of $\rho_{\varepsilon}$, but rather to clarify some relations that might be helpful when assessing the riskiness of certain financial positions in the insurance context.

The present approach is somewhat different from Dhaene et al. (2003). Apart from the fact that these authors use the Lundberg bound instead of the ruin probability itself, they fix the initial capital and determine the annual premium in their discrete-time model according to the required level of the ruin probability. Here, we fix the safety loading (and hence the premium relative to the claim rate $\lambda$ ) and try to allocate the appropriate initial capital for the required level of the ruin probability. Motivation for that approach has been given in terms of market competition.

The risk measures discussed in the present paper enjoy nice properties and appear natural in the classical framework of risk measures: they are just a VaR and a Tail-VaR of a random variable, namely the minimum $L$ of a stochastic process. Some of the results established in the present paper might be extended to general renewal models. For instance, the ruin probability is then still obtained from the tail function of a geometric compound distribution, 
although the ladder height distribution is in general not the integrated tail and not explicitly available anymore.

\section{Acknowledgements}

The authors express their gratitude to an anonymous referee and to the editor whose comments have been extremely useful to revise a previous version of the present work. Michel Denuit and Julien Trufin acknowledge the financial support of the Communaute francaise de Belgique under contract Projet d'Actions de Recherche Concertees ARC 04/09-320. Michel Denuit acknowledges the financial support of the Onderzoeksfonds K.U. Leuven (GOA/07: Risk Modeling and Valuation of Insurance and Financial Cash Flows, with Applications to Pricing, Provisioning and Solvency). Hansjoerg Albrecher acknowledges support of the Austrian Science Fund Project P18392.

\section{References}

Asmussen, S. (2000) Ruin Probabilities, Singapore: World Scientific.

Cheridito, P., Delbaen, F. and Kupper, M. (2006) 'Coherent and convex monetary risk measures for unbounded cadlag processes', Finance and Stochastics 10: 427-448.

Denuit, M., Dhaene, J., Goovaerts, M.J. and Kaas, R. (2005) Actuarial Theory for Dependent Risks: Measures, Orders and Models, New York: Wiley.

Dhaene, J., Goovaerts, M.J. and Kaas, R. (2003) 'Economic capital allocation derived from risk measures', North American Actuarial Journal 7: 44-59.

Embrechts, P., Kaufmann, R. and Samorodnitsky, G. (2004) 'Ruin theory revisited: Stochastic models for operational risk', in C. Bernadell et al. (ed) Risk Management for Central Bank Foreign Reserves, Frankfurt: European Central Bank, pp. 243-261.

Embrechts, P. and Puccetti, G. (2006) 'Aggregating risk capital, with an application to operational risk', The Geneva Risk and Insurance Review 31: 71-90.

Geman, H. (1998) 'Learning about risk: Some lessons from insurance', European Finance Review 2: $113-124$.

Heilmann, W.-R. and Schröter, K.J. (1991) Orderings of risks and their actuarial applications. Stochastic orders and decision under risk IMS lecture notes-Monograph series 19, Hayward, California, pp. 157-173.

Huang, H.-H. (2006) 'Optimal insurance contract under a value-at-risk constraint', The Geneva Risk and Insurance Review 31: 91-110.

Kaas, R., Goovaerts, M.J., Dhaene, J. and Denuit, M. (2008) Modern Actuarial Risk Theory Using $R$, New York: Springer.

Lefèvre, C. and Utev, S. (2001) 'Comparison of individual risk models', Insurance: Mathematics and Economics 28: 21-30.

Luciano, E. and Kast, R. (2001) 'A value-at-risk approach to background risk', The Geneva Papers on Risk and Insurance Theory 26: 91-115.

Michel, R. (1987) 'A partial ordering of claim amount distributions and its relation to ruin probabilities in the Poisson model', Bulletin of the Swiss Association of Actuaries 1987: $75-80$.

Pflug, G. and Römisch, W. (2007) Modeling, Measuring and Managing Risk, New Jersey: World Scientific.

Shaked, M. and Shanthikumar, J.G. (2007) Stochastic Orders, New York: Springer. 


\section{About the authors}

Julien Trufin PhD student, is a Fellow of the Royal Society of Belgian Actuaries, Institute of Actuarial Science, Université Catholique de Louvain, Louvain-la-Neuve, Belgium.

Hansjoerg Albrecher $\mathrm{PhD}$, is Professor of Actuarial Science at the Ecole des HEC (Business School), Université de Lausanne, Switzerland.

Michel Denuit PhD, is a Fellow of the Royal Society of Belgian Actuaries, Professor of Statistics and Actuarial Mathematics, Institute of Statistics, Biostatistics and Actuarial Science, Université Catholique de Louvain, Louvain-la-Neuve, Belgium. 